\title{
Technologie et design de jeu
}

\section{Jonathan Lessard}

\section{(2) OpenEdition}

Journals

Édition électronique

URL : http://journals.openedition.org/sdj/521

DOI : $10.4000 /$ sdj.521

ISSN : 2269-2657

Éditeur

Laboratoire EXPERICE - Centre de Recherche Interuniversitaire Expérience Ressources Culturelles Education

\section{Référence électronique}

Jonathan Lessard, «Technologie et design de jeu », Sciences du jeu [En ligne], 4 | 2015, mis en ligne le 05 octobre 2015, consulté le 06 avril 2021. URL : http://journals.openedition.org/sdj/521 ; DOI : https://doi.org/10.4000/sdj.521

Ce document a été généré automatiquement le 6 avril 2021.

\section{(c) (1) (8)}

La revue Sciences du jeu est mise à disposition selon les termes de la Licence Creative Commons Attribution - Pas d'Utilisation Commerciale - Pas de Modification 4.0 International. 


\title{
Technologie et design de jeu
}

\author{
Jonathan Lessard
}

1 La technologie occupe une part importante du discours sur l'évolution des jeux vidéo. Lorsqu'il évoque l'avenir du jeu sur ordinateur en 1982, le journaliste Dick McGath du magazine Computer Gaming World résume : «La plupart des attentes sont centrées sur les innovations techniques » (McGath, 1982, p. 33) ${ }^{1}$. Ses prédictions portent, entre autres, sur l'avènement de graphismes plus réalistes grâce à une meilleure définition et une plus grande finesse dans les périphériques d'entrée. Plus de 30 ans plus tard, le discours n'a à cet égard guère changé. Dans le numéro d'avril 2014 du magazine PC Gamer traitant aussi de l'avenir du jeu sur ordinateur, la technologie occupe encore l'essentiel du dossier. Les attentes portent d'ailleurs toujours sur les périphériques d'entrée et de sortie, en l'occurrence les casques de réalité virtuelle, la définition $4 \mathrm{~K}$, les contrôleurs main-libre et le tapis roulant omnidirectionnel.

2 Plusieurs des succès les plus phénoménaux du jeu vidéo ne semblent pourtant pas particulièrement liés à des innovations technologiques. Si l'on se fie aux données de la compagnie Electronic Arts, le jeu Tetris resterait l'un des jeux les plus lucratifs au monde (plus de 100 millions de ventes) (Tucker 2010) bien que la technologie nécessaire à son exécution fut disponible depuis le début des années 1980. Plus récemment, le jeu Candy Crush Saga, d'une relative simplicité technique, rapportait à son propriétaire plus d'un milliard de dollars (Shontell 2014). Une perspective plus axée sur le design offre une alternative au discours technocentriste. Dans son livre, le concepteur de jeu Richard Rouse III exprime une opinion partagée par plusieurs joueurs et designers : « des graphismes attirants ou une programmation de pointe ne compenseront jamais un design de jeu médiocre » (Rouse, 2010, p. xxiii).

3 Cette prédominance du design comme facteur d'évolution semble d'autant plus évidente dans le domaine des jeux non-numériques. Certains classiques de l'histoire des jeux de cartes (Parlett 1990) ou des jeux de société (Parlett 1999, Woods 2012), par exemple, ne se réfèrent que très peu - voire pas du tout - à des facteurs technologiques pour rendre compte des changements que connaissent leur objet d'étude. Cependant, l'invention même des cartes à jouer est associée à un contexte technologique. En effet, le philosophe et historien du jeu de tarot, Michael Dummett, souligne que ce n'est pas 
un hasard si les cartes à jouer voient le jour en Chine: "où tant le papier que l'impression ont d'abord été inventés " (1980, p. 34). Plus récemment, Elias et al. (2012, p. 81) associent l'émergence des jeux de cartes utilisant des paquets non-standards (Magic: The Gathering, par exemple) à la souplesse de l'équipement d'impression moderne.

Un coup d'œil du côté de l'histoire moderne des sports suffit pour trouver des rapports étroits entre technologie et jeux non-numériques. Certaines activités ludiques telles que les courses automobiles ou cyclistes ne dépendent pas moins d'un support technologique que les jeux vidéo. Certains sports aussi minimalistes que la natation voient leur évolution fortement influencée par la contribution d'innovations techniques. En l'occurrence, pas moins de 62 records mondiaux récents sont associés à la combinaison hydrofuge LZR Racer de Speedo (Thurow 2008).

5 C'est sans grande surprise que l'on constate l'existence de rapports plus ou moins étroits entre technologie et évolution des jeux, tant numériques que traditionnels. Il est plus surprenant que les études du jeu et de leur design, stimulées pourtant par le phénomène très technologique du jeu vidéo, ne se soient pas davantage penchées sur la nature de ces interactions. Il semble que dans leur désir de fonder une ludologie autonome et générale, certains théoriciens et designers plus « puristes » tels que Salen et Zimmerman (2003), Juul (2005), Elias et al. (2012) ou Adams et Dormans (2012) préfèrent minimiser le rôle des conditions matérielles au profit de structures plus abstraites. D'autre praticiens plus pragmatiques tels que Schell (2007), Adams (2012) ou Rouse (2010) tendent à davantage reconnaître les contraintes technologiques dans le processus de design, mais réduisent généralement celles-ci à une donnée à considérer en amont ou un instrument à façonner en aval, sans examiner les rapports dynamiques que design et technologie peuvent entretenir.

6 Cet article vise à identifier et décrire les principales modalités d'interaction entre technologie et design de jeu. Il s'inscrit dans un projet plus vaste visant à mieux comprendre les mécanismes d'évolution formelle des jeux et des processus associés à leur conception. L'approche est historique plutôt que théorique: comment la technologie a-t-elle influencée les jeux que nous connaissons? Peut-on dégager certains principes généraux de ces interactions? Afin de couvrir le plus large spectre de jeux, la démarche adoptée est celle d'une "méta-analyse ", soit une revue d'un large ensemble de sources historiques secondaires. Un corpus d'ouvrages portant sur l'histoire de différents jeux traditionnels, numériques et sports a été consulté. Compte tenu de la rareté des ouvrages académiques sur le sujet, plusieurs histoires dites «d'amateur» ont dû être mises à profit. En raison de l'ampleur et de la relative nouveauté du sujet, la présente étude se veut davantage un défrichage qu'une enquête exhaustive.

7 Nous reviendrons dans un premier temps sur les principales perspectives actuelles permettant de rendre compte des rapports qu'entretiennent design de jeu et technologie. Un modèle alternatif sera ensuite proposé, menant à une analyse des interactions entre trois pôles : technologie, design de jeux (games) et jeu (play).

\section{Technologie et jeux : au-delà de la plateforme}

8 D'un point de vue purement ludologique, les jeux peuvent être envisagés de façon abstraite, en dehors de toute matérialité et donc indépendants de facteurs 
technologiques. Salen et Zimmerman proposent dans Rules of Play que les « règles forment la structure profonde d'un jeu à partir desquelles toutes les instances réelles du jeu sont dérivées" (Salen et Zimmerman, 2003, p. 120). Pour illustrer la notion de règles constitutives, «les structures formelles sous-jacentes existant "sous la surface" des règles présentées au joueurs» (p. 132), ils décrivent ainsi le jeu de Serpents et Échelles :

1. Les joueurs commencent tous avec une valeur de 0 .

2. Chacun à leur tour, les joueurs ajoutent un chiffre aléatoire de 1à 6 à leur valeur actuelle.

3. Le premier joueur à atteindre une valeur de 100 exactement gagne (si l'ajout du chiffre aléatoire au total du joueur excèderait 100, ne pas ajouter ce chiffre aléatoire ce tour-ci).

4. Lorsque le total d'un joueur atteint certains nombres spécifiques, le total change. [...] (p. 132)

9 La dernière règle décrit le mécanisme général des "raccourcis " représentés par les serpents et échelles; les auteurs ne prennent cependant pas la peine de spécifier chaque raccourci, ce qui serait pourtant nécessaire pour rendre ces «règles constitutives » fonctionnelles.

Il y a bien une certaine correspondance entre cette description algorithmique et les Serpents et Échelles, et il serait effectivement possible d'y jouer de la sorte. Cependant, cette réalité est très éloignée de l'expérience des joueurs et, fort probablement, des concepteurs du jeu également. En effet, il est difficile d'imaginer le processus de design des Serpents et Échelles sans l'associer à la manipulation exploratoire de dés à six faces et de jetons sur une surface. Le problème pragmatique est d'autant plus criant pour les jeux numériques dont les règles constitutives, dans leur exhaustivité, sont pratiquement impossibles à dissocier de leur implémentation matérielle et logicielle.

11 Dans Half-Real, Jesper Juul (2005, p. 48), précise que : « la définition des jeux proposée ici ne rattache pas les jeux à un médium spécifique ». Juul considère les jeux comme des objets «transmédiatiques » (transmedial), c'est-à-dire qu'ils peuvent effectivement être envisagés en abstraction de leur support bien que leur incarnation dans un média ou un autre puisse modifier l'expérience de jeu. Le tennis, par exemple, existerait comme un ensemble de règles abstraites dont l'expérience effective varie selon qu'il est joué sur une Playstation ou sur un terrain avec une balle physique. Juul parle "d'implémentation » lorsqu'un jeu maintient une expérience similaire sur un nouveau média (les échecs sur ordinateur, par exemple) et "d'adaptation» lorsque les différences expérientielles sont importantes (les sports, par exemple) (Juul, 2005, p. 49). Cette perspective permet de rendre compte de la déclinaison de certains jeux sur de multiples supports mais ne permet pas une analyse technologique très précise. Quel est le média du tennis? La raquette? La balle? Le terrain? Les joueurs humains? La « vraie vie »? Et si on parvenait à l'identifier, combien d'autres « contenus » ce média supporte-t-il effectivement?

12 L'approche des «platform studies » initiées par Bogost et Montfort (2009) avec l'ouvrage Racing the Beam sur l'Atari 2600 est plus ancrée dans une histoire matérielle des jeux. Le projet en est de mieux comprendre l'influence des contraintes matérielles de certains artefacts technologiques (consoles de jeu généralement) sur le design des jeux dont ils sont le support. Cette perspective donne lieu à des découvertes intéressantes mais permet plus difficilement de rendre compte de la rétroaction du design de jeu sur le développement technologique. Bogost et Montfort (2009, p. 52) soulignent justement que l'architecture de l'Atari 2600 elle-même avait été pensée en fonction de jeux 
préexistants, notamment PONG (1972) ou Tank (1974). De même, les jeux de tir "shoot'em up " auraient plus tard une influence sur la spécification de la console PC Engine (Therrien et Picard, 2015, p. 11). Ainsi les plateformes subissent-elles autant l'influence des jeux qui les précèdent qu'elles en exercent sur ceux qui suivent. L'angle de la plateforme pose également problème pour tous les jeux n'opérant pas en fonction d'une "plateforme » entendue comme "un standard particulier ou une spécification précédant toute implémentation " (Bogost et Montfort 2009, p.7). C'est-à-dire qu'à l'exception peut-être du jeu de cartes standard et du dé à six faces, le champ des platform studies se réduit essentiellement aux jeux prenant la forme de logiciels dépendants d'une spécification matérielle.

13 Dans son Art of Computer Game Design, le designer Chris Crawford présente la technologie comme « matériau » du designer :

Toutes les formes d'art s'expriment par un médium physique. Le contrôle et la manipulation de ce médium représentent un problème technique que l'artiste doit pouvoir résoudre avant de l'utiliser pour s'exprimer à travers lui. Ainsi, le sculpteur doit pleinement comprendre les contraintes associées au marbre, au cuivre ou à tout autre matériau qu'il utilise. [...] Il en va de même pour le designer de jeux vidéo, qui doit commencer par maitriser le médium avec lequel il travaille (Crawford, 1997, p. 35).

14 Pour Crawford, ce rapport entre technologie et design de jeu ne se limite pas aux jeux d'ordinateurs puisqu'il poursuit en décrivant les affordances ${ }^{2}$ de la "technologie ludique » du paquet standard de 52 cartes. Envisager la technologie comme une matière première à laquelle le designer donne forme permet de mieux rendre compte du facteur technologique dans le design et l'évolution d'un jeu comme le tennis: la conception du «lawn tennis» est tributaire de la disponibilité de balles en caoutchouc vulcanisé, nous y reviendrons. Cependant, cette perspective ne tient compte que du rapport du designer à la technologie. Or, de nombreuses innovations techniques liées aux jeux sont issues des joueurs et de leur désir de bien performer selon les critères établis par les règles d'un jeu. Les règles générales du tennis moderne telles que stabilisées vers la fin du XIX ${ }^{\mathrm{e}}$ siècle laissaient un certain « jeu » dans l'interprétation de ce qu'est une raquette. Trouver les paramètres techniques de la raquette de tennis idéale est devenu un problème d'ingénierie menant à de nombreuses découvertes et innovations ayant à leur tour laissé leur marque sur l'expérience de ce jeu.

15 Afin de rendre compte plus finement du rôle de la technologie dans l'évolution formelle des jeux, nous tâcherons de mieux décrire les interactions entre trois pôles: technologie, design de jeu (game) et jeu (play). La technologie, une notion aussi résistante à la définition que celle de jeu, prendra ici le sens général que nos acteurs (designers et joueurs) pourraient lui attribuer dans le contexte de leurs pratiques respectives; c'est-à-dire, grosso modo, comme somme des connaissances appliquées pouvant donner forme à des structures de jeu ou aux instruments nécessaires à leur pratique. Afin d'envisager le phénomène des jeux dans sa globalité, le mot «technologie» sera employé (anachroniquement) pour désigner l'ensemble de la technè humaine, comprenant ce qui était jadis nommé «les arts», sans particulièrement distinguer la technologie moderne. Il est probable que cette approche occulte des différences significatives entre les régimes technologiques traditionnels et contemporains, mais ces nuances devront être faites dans des travaux ultérieurs.

16 La notion de "design de jeu » entendu comme processus volontaire - voire même professionnel - est également anachronique lorsqu'appliquée à de nombreux jeux 
traditionnels. Néanmoins, à des fins de cohérence et de clarté, nous l'emploierons rétroactivement pour décrire tout acte de formalisation de jeu, qu'il s'agisse de joueurs de cartes du dimanche qui décident de mettre à l'épreuve une nouvelle règle dans leur jeu préféré ou d'officiels d'une ligue sportive qui ajustent le règlement pour évacuer une forme de comportement indésirable. L'évolution tant technologique que ludique est envisagée dans la lignée des travaux sur la construction sociale de la technologie (par exemple Bijker, 1995), soit en reconnaissant la flexibilité interprétative des artefacts et leur nature socialement construite. Notons enfin qu'au cours du texte, le mot « évolution » sera employé au sens neutre de « changements dans le temps » sans impliquer une direction téléologique ou un progrès absolu.

17 Les sections qui suivent s'attardent à décrire, sur la base d'exemples historiques, les différentes interactions reliant la technologie, le design de jeu et le jeu (play). Celles-ci esquisseront les arrêtes d'un modèle triangulaire que nous présenterons plus tard dans son ensemble. Pour commencer, attardons nous à l'intuition populaire voulant que la technologie permette l'élaboration de nouvelles formes de jeux.

\section{Technologie $\rightarrow$ Design de Jeu}

18 Le discours technocentriste des magazines de jeux vidéo évoqué en introduction suggère que les nouvelles technologies engendrent de nouveaux jeux. Nous examinerons plus précisément ce mouvement en abordant le cas de jeux dont la genèse est fortement associée à la technologie. Nous commencerons par un exemple on ne peut plus éloigné du jeu vidéo, le tennis sur gazon, que nous comparerons ensuite aux premiers jeux d'aventure sur ordinateur.

Si l'on en croit plusieurs histoires du tennis, la forme moderne de ce sport serait en partie la conséquence d'une innovation technologique :

La popularité du jeu [de tennis] diminua au cours du XVIII siècle, mais connue une autre révolution en 1850 lorsque que Charles Goodyear inventa un procédé pour le caoutchouc nommé la vulcanisation, rendant le matériel utilisé pour les balles de tennis significativement plus élastique. En conséquence, le tennis pouvait maintenant être joué à l'extérieur sur la pelouse. Les fondations du tennis moderne avaient été établies (History of tennis, 2014).

20 Lorsque Charles Goodyear découvre la vulcanisation du caoutchouc au tournant des années 1840 , son objectif n'est probablement pas d'améliorer le tennis. Reste que l'élasticité des balles faites avec ce matériau semble jouer un rôle dans le design du tennis moderne.

21 En 1873, un certain major anglais du nom de Walter Clopton Wingfield conçoit, brevète et distribue un jeu de balle et raquette du nom de Sphairistikè («art de la balle» en grec). Le jeu se vend sous la forme d'une boîte comprenant filet, poteaux, raquettes, des balles de caoutchouc au centre vide et un document expliquant les règles. Aucun de ces éléments ne constitue alors une technologie nouvelle. D'ailleurs, le jeu lui-même est extrêmement proche du tennis, un sport joué depuis le moyen-âge. Au moment de la sortie du Sphairistikè, l'évolution du tennis depuis le jeu de paume avait déjà intégré l'usage de la raquette et du filet (Gillmeister, 1998).

22 Malgré ces ressemblances, le nouveau jeu propose une expérience ludique suffisamment différente du tennis traditionnel pour être vécu comme une rupture significative. Cette différence est en bonne partie due aux propriétés de la balle en 
caoutchouc vulcanisé, principalement son élasticité. Jusqu'alors, on doit s'adonner au tennis sur des surfaces suffisamment dures pour permettre aux balles de cuir ou de mastic de rebondir. La balle de caoutchouc permet au Sphairistikè de se pratiquer à l'extérieur, ce qui lui vaudra le nom plus accessible de Lawn Tennis (tennis sur pelouse). Même sur cette surface plus absorbante, la balle de caoutchouc rebondit tellement que le document accompagnateur conseille aux joueurs de frapper la balle doucement (Gillmeister, 1998, p.185). L'historien du tennis Gillmeister souligne que cette indication visait les joueurs de tennis contemporains qui avaient l'habitude de frapper aussi fort que possible ; d'une part parce que la balle rebondissait moins, d'autre part parce que le jeu étaient joué entre quatre murs qui renvoyaient la balle vers le terrain de jeu.

23 Le changement de balle et de terrain entrainent toute une série de modification au design de cette nouvelle forme de tennis. Sans murs pour renvoyer la balle, Wingfield mise sur des lignes de démarcation qui deviennent très importantes pour le tennis sur pelouse. Contrôler la position exacte du contact au sol d'une balle très dynamique devient un des enjeux majeurs de ce nouveau jeu. Le filet est aussi modifié pour circonscrire l'activité de la balle dans un environnement ouvert. Celui-ci est beaucoup plus élevé (1.5 mètre aux extrémités et 1.2 au centre). La taille réduite du court et la hauteur du filet entraine initialement des échanges aériens et un rythme relativement posé (Gillmeister, 1998, p. 185). Certaines règles très anciennes sont abandonnées, telles que le service devant rebondir sur un toit incliné et le pointage basé sur le mécanisme de la chasse ${ }^{3}$. En relativement peu de temps, les règles initiales de Wingfield sont légèrement modifiées pour établir les principales normes du tennis moderne. Que le tennis sur pelouse marque une rupture d'avec le tennis traditionnel hérité du jeu de paume est mis en évidence par la persistance de l'ancienne forme qui se donne un nouveau nom distinctif: Real Tennis (vrai tennis). Une autre preuve en est la progressive découverte d'un nouveau savoir-faire propre au tennis sur pelouse. Il faut, par exemple, attendre une dizaine d'années avant l'établissement du service par-dessus, de l'usage commun des coups coupés, brossés, des lobs et du smash; autant de techniques inconnues de l'ancien tennis (Gillmeister, 1998, pp. 197-199).

Quelle est le rôle exact de la technologie dans la conception du Sphairistikè ? Nous ne pouvons que spéculer sur le processus précis ayant mené à la formalisation de ce nouveau jeu mais tentons néanmoins d'esquisser un scénario plausible. Le major Wingfield fait partie de la bourgeoisie anglaise qui connait alors un engouement pour les activités sociales sur pelouse (rendues praticables par la récente introduction de tondeuses manuelles) et tout particulièrement le croquet. On doit également supposer que le major est un amateur de tennis tel qu'il se pratiquait alors puisqu'il juge opportun d'en actualiser la forme. Suivant l'observation de Petroski (1993) selon laquelle l'évolution des objets procède des défaillances qui leurs sont attribuées, on peut supposer que Wingfield se met à considérer que le tennis serait "mieux " s'il pouvait se jouer sur n'importe quelle pelouse plutôt que dans des salles dédiées. Peutêtre son objectif est-il plutôt (ou aussi) de rendre ce sport relativement exigeant accessible à un plus grand public.

En amont ou en aval de cette réflexion, le major met probablement la main sur une balle de caoutchouc vulcanisée telle qu'elles étaient déjà produites en Allemagne. Cette nouvelle forme de balle et ses propriétés s'ajoutent au répertoire technique dans lequel il peut puiser pour imaginer un renouvellement du tennis. Celle-ci offre à Wingfield 
une solution au problème de jouer à un jeu nécessitant qu'une balle rebondisse sur de la pelouse. Une fois cette technologie intégrée au nouveau jeu, ses propriétés agissent comme contrainte sur le reste de la formalisation: lignes, filet élevé, règles de pointage, etc. C'est là que l'on retrouve les qualités du designer de jeu : il ne suffisait pas d'ajouter une balle de caoutchouc au tennis tel que pratiqué ou de le faire sortir dehors ${ }^{4}$; le major Wingfield fait preuve, avec les règles du Sphairistikè, d'une grande sensibilité aux rapports entre mécanismes ludiques formels et esthétique de jeu.

Le caoutchouc vulcanisé a certainement joué un rôle dans le processus de design du tennis moderne. Il lui fallait cependant rencontrer un contexte social propice à l'idée que jouer au tennis sur du gazon pourrait être fun. On peut néanmoins considérer la technologie ici comme une "affordance " pour le designer : la balle en caoutchouc se prête au rebondissement, y compris sur une surface molle, et peut donc être mobilisée dans la résolution du "problème » du tennis sur gazon. D'ailleurs, peut-être est-ce l'élasticité même de cette balle qui expose les «défaillance » du tennis traditionnel. Le major Wingfield a-t-il vu en la balle de caoutchouc une solution au problème de faire rebondir une balle sur du gazon, ou celle-ci a-t-elle mis en lumière le «manque » de rebondissement de la balle de cuir traditionnelle (qui n'avait pas nécessairement été remarqué jusque là) ? Dans tous les cas, l'introduction de la balle de caoutchouc ne fait pas qu'ouvrir de nouvelles portes au designer, elle en ferme d'autres au passage. Autrement dit, la technologie n'est pas que potentiel, elle incarne également des contraintes. L'élasticité de la balle de caoutchouc permet le rebondissement, mais au détriment de toute autre alternative. Autrement dit, elle condamne au rebondissement. Dorénavant, les joueurs de tennis ne pourront plus frapper de toute leur force à moins de parfaitement maitriser la trajectoire et la rotation de la balle. Les nouvelles règles concernant les lignes, le service et le filet sont autant d'efforts pour "dompter » l'élasticité de la balle du caoutchouc et la rendre compatible avec l'idée que l'on a alors du tennis.

27 Voyons si ces observations tiennent dans le cas d'une technologie plus récente que la vulcanisation du caoutchouc. L'invention du jeu d'aventure sur ordinateur présente un cas dont la genèse est mieux documentée (Lessard 2013b). William Crowther, le concepteur de la première version d'Adventure (1976), a explicitement exprimé qu'il s'agissait d'une tentative d'adapter Dungeons \& Dragons (1974) pour l'ordinateur. Cette technologie est donc au cœur du projet et les modalités de l'informatique de l'époque suggèrent et contraignent à la fois les formes possibles de l'éventuel jeu. Par exemple, le « maître du donjon ", normalement joué par un humain, y prend la forme d'une base de données de descriptions pré-écrites avec laquelle un joueur interagit par le biais de commandes textuelles simples. Ce programme obligeant les joueurs à découvrir par essai et erreur les phrases exactes qui leur permettront de progresser dans un labyrinthe de cavernes décrites textuellement, est bien loin de la souplesse et de l'improvisation collective de son modèle.

$28 \mathrm{Vu}$ sous cet angle, il est difficile de défendre que la technologie incarnée par l'ordinateur a permis d'améliorer le jeu de rôle. L'informatique offre bien des affordances, mais c'est davantage ses contraintes que cet exemple met en lumière: sourd, muet, idiot et dénué d'imagination, l'ordinateur fait un bien piètre maître du donjon. Ce jugement est cependant injuste; le problème de design auquel s'attaque Crowther n'est pas tant d'améliorer l'expérience du jeu de rôle, mais plutôt celle de l'ordinateur lui-même qu'il aimerait rendre plus accessible à ses enfants. De cette 
collision entre le design du jeu de rôle et les affordances et contraintes de l'ordinateur émerge une forme ludique nouvelle; celle-ci permettant à des non-techniciens d'interagir avec un ordinateur pour résoudre plaisamment des "puzzles de fiction " (Karhulahti, 2014) à leur propre rythme, sans avoir à intégrer et s'adapter à un groupe de jeu (comme c'est le cas pour Donjons et Dragons).

29 S'il est évident que la technologie rend possible certaines formes de jeux qui ne pourraient être autrement envisageables, celle-ci ne peut être considérée simplement comme un instrument au service du design, un réservoir de potentiel. Envisagée comme "matériau», la technologie agit aussi bien comme affordance que comme contrainte et fait autant partie de la définition des problèmes auxquels s'attaque le design que de leur solution. Ces rapports ne sont évidemment pas unidirectionnels et nous nous pencherons dans la section suivante sur la rétroaction du design de jeu sur le développement technologique.

\section{Design de Jeu $\rightarrow$ Technologie}

Si Adventure a été pensé avec l'ordinateur comme donnée technologique du problème, le casque de réalité virtuelle est une technologie qui, de toute évidence, est motivée par la défaillance perçue des jeux actuels qui ne seraient pas encore assez "immersifs ». L'argumentaire de la campagne initiale de sociofinancement de l'Oculus Rift est assez limpide : « le Rift permet une expérience réellement immersive permettant d'entrer au sein de votre jeu favori et d'explorer de nouveaux mondes comme jamais auparavant " (Oculus, 2012). Ce discours masque bien sûr le lot de contraintes nouvelles que ce gadget importera dans l'écologie des jeux mêmes qu'il souhaite » augmenter ». On voit pourtant déjà que les jeux de tir à la première personne ne pourront pas être joués tels quels au risque de voir les joueurs pris immédiatement de nausées; ou que le standard d'interaction bien établi du duo clavier et souris n'est pas compatible avec un contexte de réalité virtuelle.

31 Mêmes les artefacts technologiques conçus expressément pour répondre à des problèmes de design de jeu n'agissent pas comme pures affordances mais également comme contraintes et il s'établit entre ces deux pôles une boucle de rétroaction constructive. En effet, les contraintes technologiques sont tout aussi productives du point de vue de l'évolution du design de jeu que leurs potentialités. Le jeu d'aventure n'existerait pas si l'ordinateur pouvait effectivement remplacer un joueur humain et le tennis moderne doit autant à l'aspect incontrôlable de la balle en caoutchouc qu'à sa capacité à rebondir sur du gazon. D'ailleurs, l'un des jeux les plus remarqués pour l'Oculus Rift à ce jour est Dumpy: Going Elephants (Schrank 2014) qui, loin de réaliser le fantasme "d'entrer dans le jeu », exploite habilement le détecteur de mouvements de la tête du casque pour mettre le joueur au défi d'utiliser efficacement une trompe d'éléphant. Un des critiques relève justement que ce design apporte une solution ingénieuse à l'un des problèmes introduits par cette technologie: «[ce jeu] résout élégamment le problème d'absence de contrôleur de l'Oculus Rift " (Dumpy: Going Elephants for Oculus Rift, 2013).

Les exemples de problèmes technologiques définis par un projet de design ludique abondent dans les sports. Dans son étude de l'évolution de la bicyclette, l'historien des sciences Wiebe E. Bijker affirme: «Alors que le ski a commencé comme façon de se déplacer et a évolué en un sport, le cyclisme commença comme une activité sportive et 
a évolué en moyen de transport» (Bijker, 1995, p. 37). La construction sociale initiale du bicycle comme objet ludique permet d'expliquer son évolution formelle vers le grand-bi, une machine parfaitement adaptée pour constituer ce que l'on appellerait aujourd'hui un "sport extrême ", mais beaucoup trop dangereuse et difficile à opérer pour être une option de transport viable pour le grand public. Quant aux skis, s'ils ont d'abord été développés comme moyen de transport, ceux-ci sont devenus une affordance pour le design de jeu qui a ensuite poussé leur développement technologique dans de nouvelles directions. Dans son histoire technologique du ski, Gertrud Pfister (2001) montre comment des skis particuliers sont développés pour accommoder la diversification des pratiques : longueur, courbe, matériau, adhérence et fixations sont autant de paramètres techniques développés et ajustés aux différents projets ludiques que sont le ski alpin, le ski de fond, le saut à ski, etc.

L'histoire de l'escrime montre bien que le design de jeu et le développement technologique établissent des rapports de rétroaction complexes dont émergent des formes qui dépassent les intentions des acteurs. Par exemple, les introductions successives du fleuret comme arme de pratique $\left.(\mathrm{au} \mathrm{XVI})^{\mathrm{e}}\right)$ ainsi que du masque à treillis $\left(\mathrm{au} \mathrm{XIX}^{\mathrm{e}}\right)$ et du contrôle électrique des touches (au tournant du XX) visent toutes à supporter le projet ludique général de l'escrime, soit de permettre une simulation de combat à l'épée sans risque. Ce qu'on observe cependant, et qui semble former un pattern, c'est que le projet ludique ne se sort pas indemne de ces innovations technologiques volontaires. L'introduction d'une arme plus légère et plus souple qu'une épée réelle, d'un masque protégeant le visage et éventuellement d'un contrôle des touches précis au centième de seconde entraîne l'émergence d'une escrime sportive, d'un jeu acrobatique au rythme effréné n'ayant que très peu à voir avec le vrai duel (Six, 2007). Ce dernier exemple montre bien que les interactions entre les designers et la technologie de suffisent pas à brosser un tableau complet; en s'appropriant les innovations techniques de l'escrime et en explorant leurs propriétés, les joueurs jouent également un rôle dans son évolution formelle.

\section{Technologie $\leftrightarrow$ Jeu (play) $\leftrightarrow$ Design de Jeu}

Dans une analyse sociologique de l'évolution des jeux, Francis Kew (1987) met en lumière la présence de deux parties prenantes aux objectifs contradictoires: les "rulemakers» (que nous entendrons comme « designers de jeu»), dont l'objectif est de définir et maintenir la cohérence du projet ludique ; et les joueurs qui, ayant accepté ces contraintes, dirigent toutes leurs actions vers l'objectif de gagner. Les efforts des joueurs tendront donc vers la découverte de stratégies dominantes, quitte à mettre en péril l'équilibre actuel du jeu.

Le design d'un jeu génère des problèmes inédits ; par exemple comment renvoyer une balle en caoutchouc avec un maximum de puissance tout en la maintenant à l'intérieur des lignes? Face à ces défis, les joueurs développent des solutions qui sont de l'ordre du développement d'heuristiques, tactiques, stratégies, d'habiletés motrices ou de techniques du corps : brosser la balle, sauter de dos plutôt que de face (le Fosbury Flop), occuper le milieu de l'échiquier, se constituer une courte au bridge, etc. Il arrive aussi que les joueurs mobilisent ou stimulent le développement d'une technologie» externe» au jeu pour améliorer leurs performances. Ce «jeu technologique » des joueurs n'est pas sans impact sur les structures elles-mêmes. 

complexes. Les compétitions sportives internationales mettent une énorme pression sur les joueurs qui se tournent vers la technologie pour trouver des expédients leur permettant d'améliorer leurs performances. Ceux-ci sont perçus comme contraires à «l'esprit» du jeu, et des règles sont ajoutées (design) spécifiant que leur usage est interdit. Cette situation crée le nouveau problème technologique du stéroïde anabolisant indétectable, qui lui-même stimule de nouvelles mesures de contrôle, etc. À ce point on pourrait considérer que les athlètes ne "jouent " plus puisqu'ils entrent plutôt dans le domaine de la triche. On peut cependant retrouver cette dynamique dans des comportements de bonne foi.

Nous avons évoqué l'évolution de la raquette de tennis en introduction. Celle-ci, associée au perfectionnement des joueurs professionnels, a entrainé une incroyable accélération des services. Selon le spécialiste Andy Miah (2000), les services actuels approchant les $225 \mathrm{~km} / \mathrm{h}$ arrivent au seuil de la capacité humaine à effectivement renvoyer la balle. Ces performances transforment peu à peu l'expérience du tennis même qui tend à ne devenir qu'un sport de service plutôt que d'échanges. Miah rapporte l'inquiétude de la Fédération qui a envisagé de rétablir «l'essence » du sport en testant de nouveaux prototypes de balles qui ralentiraient le jeu.

Cette tension entre l'amélioration des instruments de jeu et la préservation de l'expérience ludique est également apparente au golf. L'évolution de l'équipement des balles et clubs répond généralement au désir des joueurs de frapper plus loin. Cette surenchère peut sembler contre-productive du point de vue du design de jeu. Si le but était d'en finir au plus vite, pourquoi vouloir jouer au golf? L'historien du golf Robert Browning (1985, p. 142) résume ainsi l'effet de cette évolution : «le seul résultat étant de forcer les golfeurs à marcher plus pour frapper moins ». On note, parmi les réactions des "designers", la complexification des trous, amincissant ou contorsionnant le fairway, ajoutant des pièges tels les bunkers, le tout pour empêcher que le golf ne devienne un simple concours de distance de frappe. Les règles concernant la validité des clubs sont également fréquemment révisées, comme ce fut le cas récemment pour interdire les encoches en « $\mathrm{V}$ » sur la surface de frappe, jugées trop performantes par rapport à la forme traditionnelle en carré (Hardman, 2002).

Les joueurs de jeu vidéo ne sont pas en reste lorsqu'il est question de mobiliser la technologie au service de leurs performances. Si les consoles à l'équipement à peu près standardisé laissent moins de place au «jeu technologique», la souplesse et la modularité des ordinateurs personnels ouvrent un grand champ d'expérimentation. La qualité du matériel et des périphériques tels que claviers, souris, processeurs ou cartes graphiques peut faire une différence, surtout pour les joueurs experts. Au niveau logiciel, les joueurs peuvent développer toutes sortes d'outils auxiliaires. Certains, comme les robots jouant à la place du joueur (bots) pour récolter or, expérience et équipement rare dans la série Diablo, par exemple, sont généralement considérés comme une forme de triche comparable à l'usage des stéroïdes et stimule surtout des réponses de l'ordre de la régulation. Les mods visant à améliorer un problème perçu dans l'interface peuvent parfois être considérés comme nuisibles à l'équilibre du jeu mais certains deviennent si populaires qu'ils finissent pas être intégrés au design. Par exemple, la flèche virtuelle indiquant la destination de la prochaine quête dans World of Warcraft a d'abord fait son apparition dans différents addons (mod pour ce jeu 
spécifique) développés par des joueurs pour ensuite être intégrée à l'interface officielle lors d'une mise à jour.

Ainsi le rôle de la technologie sur l'évolution des jeux ne passe pas uniquement par l'initiative des concepteurs mais aussi par l'interaction complexe entre leurs efforts pour préserver ou faire évoluer les jeux et ceux déployés par les joueurs pour gagner. L'émergence du "putt» comme aspect fondamental du golf est un autre exemple positif non-numérique de ce type de processus. Celui-ci commence par l'introduction de la balle de gutta-percha au milieu du XIX ${ }^{e}$ siècle permettant de frapper non seulement plus loin mais qui, contrairement aux balles de cuir rembourrées de plumes, roule droit lorsque la surface n'est pas trop accidentée. Cette propriété encouragea l'usage de clubs au loft très fermé (qui évolueront en putters) pour tenter des coups d'approche roulés. Reconnaissant l'intérêt de cette phase de jeu, les designers de terrains développèrent le "green ", une zone de pelouse extrêmement rase autour du trou favorisant ce jeu de précision (Browning 1985, p. 145).

\section{Un modèle des interactions entre technologie et design de jeu}

La reconnaissance du rôle des joueurs dans le circuit liant technologie et design de jeu complète l'ébauche d'un modèle permettant d'envisager les principales boucles de rétroaction liant la technologie à l'évolution formelle des jeux (Figure 1).

Figure Interactions entre technologie, design de jeu et jeu (play)

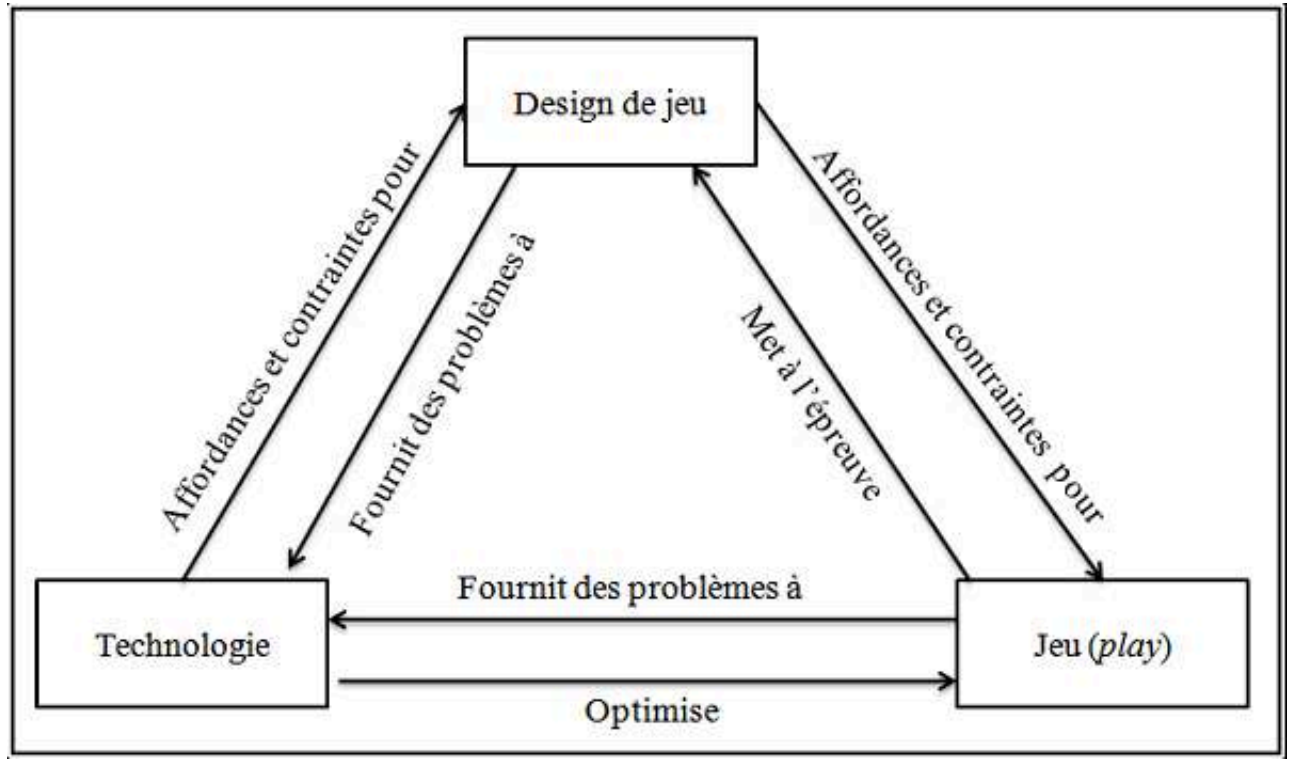

Nous avons déjà illustré par des exemples les différents vecteurs le constituant mais voyons comment celui-ci peut être mobilisé dans son ensemble pour rendre compte d'une transformation ludo-historique : le passage du jeu d'aventure textuel à la forme "pointer et cliquer». Cette histoire peut être racontée comme l'épanouissement progressif d'une forme grâce au développement technologique : capacité graphiques des ordinateurs, souris, CD-ROM, etc. Une analyse des interactions entre technologie, design et jeu brosse un tableau plus complexe. 
$43 \mathrm{Au}$ tournant des années 1980, l'affichage graphique devient plus accessible à l'informatique domestique. Cette affordance technologique est rapidement mobilisée pour pallier la défaillance nouvellement perçue des jeux d'aventures : leur absence de graphismes. L'adjonction d'illustrations rudimentaires à un style de jeu encore largement textuel permet aux designers de transférer une partie de la description du texte à l'image. Plutôt que d'explicitement mentionner : « il y a ici une roche », celle-ci est illustrée à l'écran et le joueur doit comprendre qu'il pourrait interagir avec cette roche. Sauf que la faible résolution des écrans rend cette opération de reconnaissance difficile et plusieurs joueurs se plaignent de perdre un temps fou à tenter d'identifier textuellement certains amas de pixels ambigus. Tant les designers que les joueurs se tournent vers la technologie pour résoudre ce problème. Les cartes graphiques s'améliorent sans résoudre le problème de façon satisfaisante.

Au même moment, le Macintosh fait connaître le paradigme d'interaction par interface graphique. Cette technologie est mobilisée par des designers de jeu d'aventure pour atténuer le problème (pour certains) d'avoir à "deviner» ce que le jeu peut comprendre. L'affordance de cette technologie est de rendre les verbes et objets interactifs visibles et cliquables, retirant au joueur le fardeau d'avoir à produire ses propres hypothèses. La contrainte de cette technologie est de restreindre le jeu aux objets visibles en excluant explicitement tout ce qui n'est pas à l'écran. Les joueurs testent les limites de ce nouveau système et élaborent de nouvelles stratégies mettant à mal l'expérience traditionnelle du jeu. La résolution des puzzles de fiction, qui consistait traditionnellement à tester des hypothèses jusqu'à l'épiphanie, dégénère en "chasse au pixel» (cliquer partout dans l'écran pour en découvrir les portions interactives) et en épuisement systématique des combinaisons désormais rendues explicites. Ces pratiques mettent à nouveau les designers sur la sellette alors qu'ils doivent répondre aux nouvelles critiques des joueurs trouvant désormais les nouveaux jeux trop courts ou trop faciles ${ }^{5}$.

45 Encore une fois, la mobilisation technologique pour "augmenter » le jeu d'aventure textuel (en fonction de la frustration des joueurs dans leur performance) contribue également à contraindre les possibles de la nouvelle forme qui en émerge. Le jeu " pointer et cliquer » n'est plus tout à fait le jeu d'aventure qui l'a inspiré et les joueurs trouvent dans ses affordances et contraintes de nouvelles façons de le «briser», exerçant par le fait même une pression renouvelée sur le design. Cette brève analyse permet de se dégager d'une notion de progrès technologique voulant que le jeu d'aventure pointer et cliquer est une version technologiquement améliorée du simple jeu d'aventure graphique; lui-même représentant un stade plus «évolué» de l'aventure textuelle.

\section{Conclusion}

Le sujet à l'étude étant à la fois complexe, touffu et encore sous-étudié, nous ne pouvons prétendre à des conclusions définitives et généralisables sur l'ensemble des jeux et de leur histoire. Cependant, au-delà du modèle théorique proposé, cette discussion aura contribué à réévaluer certains lieux communs concernant les rapports entre technologie et design de jeu. En premier lieu, il apparait clairement que la technologie ne détermine pas l'évolution des jeux et que celle-ci est mobilisée (ou pas) largement en fonction de questions sociales et contextuelles, et agit autant comme 
affordance que comme contrainte dans leur élaboration formelle. De même, la perspective d'une technologie entièrement au service d'un design divorcé de ses conditions matérielles ne tient pas la route. Lorsqu'un projet ludique stimule le développement spécifique d'un artefact technologique, ce dernier viendra mêler les cartes en venant lui-même (avec sa réalité matérielle obtuse) redéfinir le problème de design tel qu'initialement envisagé. Tenir compte des interactions complexes entre design de jeu et technologie permet une granularité plus fine dans l'analyse que la notion de plateforme ou du rapport média/contenu.

Finalement, il semble clair que l'activité des designers ne suffit pas à rendre compte de cette dynamique et qu'il faut y réintroduire les joueurs eux-mêmes. Ceux-ci mobilisent également la technologie, non pas dans le but de formuler un jeu mais plutôt afin de tester les limites de celui dans lequel ils sont investis. Ce faisant, ils menacent de le déstabiliser, reformulant ainsi le problème de design dont les solutions peuvent autant mener à une stabilisation renouvelée qu'à une mutation. Ces changements sont à même de favoriser de nouvelles formes de jeu (play) ou de créer de nouveaux problèmes techniques; et la roue des influences réciproques tourne, continuant d'alimenter le processus d'évolution des formes ludiques.

Rappelons toutefois que les interactions avec la technologie ne forment que l'un des moteurs de changement du jeu. Comme il apparait clairement dans certains des exemples rapportés, d'autres facteurs d'ordre culturel, social ou économique jouent un rôle tout aussi important. S'il a ici été présenté en autonomie, le modèle des interactions entre technologie et design de jeu est à envisager comme rouage d'un circuit plus large qui reste à préciser.

\section{BIBLIOGRAPHIE}

ADAMS E., \& DORMANS J. (2012), Game Mechanics : Advanced Game Design. 1st ed. Berkeley, CA : New Riders.

ADAMS E. (2010), Fundamentals of Game Design. 2nd ed. Berkeley, CA : New Riders.

BIJKER W. E. (1995), Of Bicycles, Bakelites, and Bulbs : Toward a Theory of Sociotechnical Change. Inside technology. Cambridge, MA : MIT Press.

BROWNING R. (1985). A History of Golf (Reprint edition). Mattituck, NY : Amereon Ltd [1955].

CARDELLICCHIO C. (2014), « Evolution for Games ». Board Game Studies, nº 8. http://bgsj.ludusopuscula.org/.

CRAWFORD C. (1997) The Art of Computer Game Design. Édition numérique. Washington State University Vancouver [1984]. http://pdf.textfiles.com/books/cgd-crawford.pdf.

Dumpy : Going Elephants for Oculus Rift. (2013), Digital Tools. http://www.digital-tools-blog.com/ games/3910-dumpy-going-elephants-for-oculus-rift.

DONOVAN T. (2010), Replay: The History of Video Games. Lewes :Yellow Ant. 
DUMMETT M, \& MANN S. (1980), The Game of Tarot : From Ferrara to Salt Lake City. London : Duckworth.

ELIAS G. S., GARFIELD R. \& GUTSCHERA K. R. (2012). Characteristics of Games. Cambridge, MA : MIT Press.

The Future of PC Gaming (2014), PC Gamer, April.

FULLERTON T. (2008), Game Design Workshop : A Playcentric Approach to Creating Innovative Games. $2^{\text {nd }}$ ed. Burlington, MA : Morgan Kaufmann.

GILLMEISTER H. (1998), Tennis : A Cultural History. London : Leicester University Press.

HARDMAN A. (2002), « Evaluating Changing Sport Technology : An Ethnocentric Approach ». In Sport technology : history, philosophy and policy, A. Miah et S. Eassom (eds), Oxford : Elsevier Science, pp. 135-55.

History of Tennis (2014), http://www.history.co.uk/study-topics/history-of-football-tennis/ history-of-tennis.

JUUL J. (2005), Half-Real : Video Games Between Real Rules and Fictional Worlds. Cambridge, MA : MIT Press.

KARHULAHTI V.-M. (2014), « Fiction Puzzle : Storiable Challenge in Pragmatist Videogame Aesthetics. » Philosophy and Technology 27, $\mathrm{n}^{\circ}$ 2, pp. 201-20.

KEW F. (1987), « Contested Rules : An Explanation of How Games Change ». International Review for the Sociology of Sport 22, n 2, pp. 125-35.

LESSARD J. (2013a), Histoire formelle du jeu d'aventure sur ordinateur (le cas de l'Amérique du nord de 1976-1999). Ph. D. Études cinématographiques, Université de Montréal.

LESSARD J.(2013b), « Adventure Before Adventure Games A New Look at Crowther and Woods's Seminal Program. » Games and Culture 8, n 3, pp. 119-35.

LESSARD J. (2014), « Game Genres and High-Level Design Pattern Formations. » In Third Workshop on Design Patterns in Games, colocated with the 9th International Conference on the Foundations of Digital Games. Florida.

MCGATH D. (1982). Route 80. Computer Gaming World, 2(6), pp. 33.

MIAH A. (2000), «"New Balls Please” : Tennis, Technology, and the Changing Game ». In Tennis, Science, and Technology, S.J. Haake et A.O. Coe (eds), London : Blackwell Science, pp. 285-92.

MONTFORT N., \& BOGOST I. (2009), Racing the Beam : The Atari Video Computer System. Cambridge, MA : MIT Press.

OCULUS. (2012), « Oculus Rift : Step Into the Game. » Kickstarter.. https://www.kickstarter.com/ projects/1523379957/oculus-rift-step-into-the-game.

PARLETT D. (1990), The Oxford Guide to Card Games. Oxford : Oxford University Press.

PARLETT D. (1999) Oxford History of Board Games. Oxford : Oxford University Press.

PETROSKI H. (1993), The evolution of useful things. New York : A. Knopf.

PFISTER G. (2001), « Sport, Technology and Society : From Snow Shoes to Racing Skis ». Culture, Sport, Society $4, \mathrm{n}^{\circ} 1, \mathrm{pp} .73-98$.

ROUSE R. III. (2010), Game Design : Theory and Practice, Second Edition. Burlington, MA : Jones \& Bartlett Learning. 
SALEN K., \& ZIMMERMAN E. (2003), Rules of Play : Game Design Fundamentals. Cambridge, MA : The MIT Press.

SCHELL J. (2008), The Art of Game Design a Book of Lenses. Amsterdam/Boston : Elsevier/Morgan Kaufmann.

SHONTELL A. (2014), « Candy Crush Is Doing More Than \$ 1 Billion In Sales ». Business Insider, 18 février. http://www.businessinsider.com/kingcom-generates--1-billion-from-candycrush-2014-2.

SIX G. (2007), L'invention de l'escrime. Paris: Les Quatre Chemins.

SUITS B. \& HURKA T. (2005), The Grasshopper : Games, Life and Utopia. Peterborough, Ont. : Broadview Press.

THERRIEN C., \& PICARD M. (2015), « Enter the Bit Wars : A Study of Video Game Marketing and Platform Crafting in the Wake of the TurboGrafx-16 Launch. » New Media \& Society, pré-publié en ligne le 29 avril 2016.

http://nms.sagepub.com/content/early/2015/04/29/1461444815584333.abstract

THUROW R., \& RHOADS C. (2008), « Fast Times ». Wall Street Journal, 15 août, sect. Leisure/ Weekend. http://www.wsj.com/articles/SB121865005500237497.

TUCKER L. (2010), « Tetris Game Surpasses 100 Million Paid Mobile Downloads - EA News », 21 janvier. http://www.ea.com/news/tetris-game-surpasses-100-million-mobile-downloads.

WOODS S. (2012), Eurogames : The Design, Culture and Play of Modern European Board Games. Jefferson, NC : McFarland, 2012.

\section{NOTES}

1. À moins de mention contraire, les traductions françaises de citations anglophones sont de l'auteur.

2. Le terme "affordance » sera employé pour désigner les usages perçus auxquels se prête un objet de par sa forme spécifique. Il est préféré à la traduction possible de "potentialité » en raison de son usage courant dans le discours du design, mais également pour éviter de suggérer un certain déterminisme de ce « potentiel ». L'affordance se construit dans la relation entre un objet et son utilisateur.

3. La chasse est la position exacte du $2^{\mathrm{e}}$ rebond qui est marquée et devient une cible à dépasser pour compter un point.

4. On sait d'ailleurs que plusieurs joueurs de tennis contemporains expérimentaient déjà des balles de caoutchouc sur la pelouse.

5. Tout ce processus est ici synthétisé à l'extrême, voir Lessard 2013a pour plus de détails sur l'évolution formelle du jeu d'aventure. 


\section{RÉSUMÉS}

La technologie est au cœur du discours sur l'évolution des jeux; des jeux vidéo, en premier lieu, mais également de nombreux sports. Les études du jeu actuelles ne rendent pourtant pas bien compte des interactions entre design de jeu et technologie. Cette dernière est généralement réduite aux notions de média ou de plateforme, qui impliquent des artefacts standardisés médiatisant des structures de jeux abstraites. Un tel point de vue ne rend compte ni de l'influence rétroactive du design de jeu sur la technologie, ni de la matérialité du processus de design, ni de la mobilisation de la technologie par les joueurs. Dans cet article nous proposons un modèle d'une plus fine granularité, mettant en lumière les rapports dynamiques entre design de jeu, technologie et le jeu (play) des joueurs. Ce modèle est fondé sur une méta-analyse historique de l'évolution de plusieurs jeux numériques et non-numériques.

Technology is at the heart of public discourse on game evolution-digital games, of course, but many sports as well. Yet, current game studies have not finely addressed the interactions between game design and technology. The latter is often reduced to notions of media or platform, implying standardized artefacts mediating abstract game structures. These perspectives account neither for the reciprocal influence of game design on technology, the materiality of the design process, or players' mobilization of technology in play. This article proposes a framework of finer granularity highlighting the dynamic interplay between game design, technology and play. This model is founded on a historical meta-analysis of numerous digital and non-digital games.

\section{INDEX}

Mots-clés : jeu, design, technologie, plateforme, média, histoire

Keywords : game, design, technology, platform, media, history

\section{AUTEUR}

JONATHAN LESSARD

Université Concordia 